\title{
Adropin Alleviates Myocardial Fibrosis in Diabetic Cardiomyopathy Rats: A Preliminary Study
}

\author{
Mao Liu ${ }^{1+}$, Jiao $\mathrm{Ai}^{2+}$, Zhuang Shuai ${ }^{1}, \mathrm{Kai}_{\mathrm{Tang}}{ }^{3}$, Zongyu $\mathrm{Li}^{1}$ and Yin Huang ${ }^{4 *}$ \\ ${ }^{1}$ Department of Cardiology, Cardiovascular Research Center, Affiliated Hospital of North Sichuan Medical College, \\ Nanchong, China, ${ }^{2}$ Department of Rheumatology, Affiliated Hospital of North Sichuan Medical College, Nanchong, China, \\ ${ }^{3}$ Department of Cardiology, Suining Central Hospital, Suining, China, ${ }^{4}$ Department of Cardiology, The Fifth Affiliated Hospital \\ of Sun Yat-sen University, Zhuhai, China
}

OPEN ACCESS

Edited by:

Xiaodong Zhuang,

The First Affiliated Hospital of Sun

Yat-sen University, China

Reviewed by:

Changzhi Liu,

Southern Medical University, China

Yan Li,

Jinan University, China

*Correspondence:

Yin Huang

huangyin3@sysu.edu.cn

tThese authors have contributed equally to this work

Specialty section: This article was submitted to

Cardiovascular Metabolism, a section of the journal Frontiers in Cardiovascular Medicine

Received: 31 March 2021 Accepted: 23 June 2021 Published: 12 July 2021

Citation:

Liu M, Ai J, Shuai Z, Tang K, Li Z and Huang Y (2021) Adropin Alleviates

Myocardial Fibrosis in Diabetic Cardiomyopathy Rats: A Preliminary

Study.

Front. Cardiovasc. Med. 8:688586. doi: 10.3389/fCVm.2021.688586
Aim: Adropin (ADR) is a novel regulatory polypeptide and has important effects on energy metabolism in the heart. However, it is still unclear whether ADR can relieve ventricular remodeling in DCM. Therefore, this study was conducted to assess the effect of ADR on myocardial fibrosis in DCM rats.

Materials and Methods: Twenty Wistar rats were randomly assigned into four groups: healthy control group (CON), DCM model group (DCM), DCM model treated with ADR group (ADR) and DCM model treated with perindopril group (PER). Collagen volume fraction (CVF) and perivascular collagen area (PVCA) were calculated. Diastolic function was assessed by echocardiography. The mitochondrial membrane potential assay was conducted by Rhodamine 123 staining. The protein expression levels of Col I, Col III, Mitofusin-1, Mitofusin-2 and Drp1 were evaluated using western blot.

Results: Compared to CON group, CVF, PVCA and the relative protein expression of Col I, Col III and Drp1 increased in DCM group. And the relative expression of Mitofusin-1 and Mitofusin-2 proteins decreased. During our investigations, CVF, PVCA and the relative protein expression of Col I, Col III and Drp1 decreased in ADR treated rats compared to DCM group. The diastolic function was elevated in ADR group. The fluorescence of Rhodamine 123 and the expression of Mitofusin-1 and Mitofusin-2 also increased in ADR group.

Conclusion: Our study demonstrated that ADR could alleviate myocardial fibrosis and improve diastolic function in DCM rats. ADR may be a putative candidate for the treatment of DCM.

Keywords: myocardial fibrosis, adropin, mitochondrial dynamics, mitofusin, diabetic cardiomyopathy

\section{INTRODUCTION}

Diabetic cardiomyopathy (DCM) is a type of cardiovascular damage and recognized as a specific entity in patients with type 2 diabetes mellitus, excluding coronary atherosclerotic heart disease, hypertrophic cardiomyopathy, hypertensive heart disease and other heart diseases $(1,2)$. Metabolic disturbances, myocardial fibrosis, small vessel disease and autonomic dysfunction are associated with the development of DCM. The number of deaths caused by diabetes mellitus and its complications is as high as 1.5 to 5 million each year. In addition, more than half of diabetes mellitus related fatal or disabling events are caused by DCM (3). 
Mitochondria play a pivotal role in cellular energy transduction. One-third of the heart's volume is composed of mitochondria alone as they are essential organelles in the production of adenosine triphosphate from the oxidation of fatty acid and glucose (4). Mitochondrial dysfunction may contribute to the development of DCM by inducing insulin resistance in skeletal muscle, adipose tissue and pancreatic $\beta$-cells (5).

Recent studies have found that Adropin (ADR) is closely related to cardiovascular disease (6-8). ADR, a regulatory polypeptide involved in energy homeostasis, is composed of 76 amino acids and encoded by genes related to energy balance. It is mainly expressed in tissues such as the heart, liver and brain. Besides, it is also expressed in coronary arteries and umbilical veins $(9,10)$.

ADR can regulate lipid metabolism and insulin resistance. ADR-treated mouse hearts showed improved cardiac efficiency and enhanced insulin signaling compared to control hearts. ADR-treatment protocols in vivo and ex vivo induced a reduction in the inhibitory phosphorylation of pyruvate dehydrogenase and the protein levels of the responsible kinase pyruvate dehydrogenase kinase 4 . These results indicated that ADR has important effects on energy metabolism in the heart (11). ADR can also reduce the risk of atherosclerosis and myocardial ischemia/reperfusion injury by activating the RISK signal transduction pathway $(12,13)$. Therefore, ADR may be a putative candidate for the treatment of cardiac disease associated with impaired insulin sensitivity $(7,11,14)$.

However, it is still unclear whether ADR can relieve ventricular remodeling by regulating mitochondrial dynamics in DCM. Therefore, the present study was conducted to assess the effect of ADR myocardial fibrosis in DCM rats.

\section{MATERIALS AND METHODS}

\section{Subjects}

Twenty Wistar rats were purchased from Model Animal Research Center of Nanjing University (SCXK Su. 2015-0001). All rats were 8 weeks of age at the beginning of the study. The rats were housed individually under room temperatures with 12: $12 \mathrm{~h} \mathrm{light/dark}$ cycle and free access to food and water for the duration of the study. All experimental procedures were approved by Ethics Committee of North Sichuan Medical College and complied with the guidelines for the Care and Use of Laboratory Animals.

\section{Experimental Design}

The rats were randomly assigned into four groups: healthy control group (CON), DCM model group (DCM), DCM model treated with ADR group (ADR) and DCM model treated with perindopril group (PER). In CON group, rats were given normal saline gavage $(1 \mathrm{ml} / 100 \mathrm{~g} / \mathrm{d})$ for 4 weeks without modeling operation. In DCM group, rats were given normal saline gavage $(1 \mathrm{ml} / 100 \mathrm{~g} / \mathrm{d})$ for 4 weeks after modeling operation. In ADR group, rats were given ADR gavage $(5 \mu \mathrm{g} / 100 \mathrm{~g} / \mathrm{d})$ for 4 weeks after modeling operation (15). In PER group, rats were given PER gavage $(200 \mu \mathrm{g} / 100 \mathrm{~g} / \mathrm{d})$ for 4 weeks after modeling operation (16). After the intervention, all rats were sacrificed with cervical dislocation.

\section{DCM Rat Modeling}

One-time intraperitoneal injection of $1 \%$ streptozotocin at a dose of $70 \mathrm{mg} / \mathrm{kg}$ was used to destroy the function of pancreatic $\beta$ cells. The rats' tail vein blood samples were taken on the third and seven day after the injection to test the fasting blood glucose. If both the blood glucose were more than $16.7 \mathrm{mmol} / \mathrm{L}$, and the rats had polydipsia, polyphagia and polyuria, a rat model of DCM was established $(17,18)$.

\section{Echocardiography}

Two-dimensional and Doppler echocardiography was performed to assess diastolic function using a GE Vivid 7 echocardiograph with a $10-\mathrm{MHz}$ phased array probe. Ratio of peak velocity of early (E) to late atrial (A) mitral flow (E/A) was detected. Circumferential Strain rate in early (SRe) and late (SRa) diastole were obtained from parasternal short-axis gray-scale images.

\section{Left Ventricular Weight/Body Weight Ratio}

Body weights were measured before the rats were sacrificed. The left ventricle was freed immediately after the heart was taken. An electronic balance was used to weigh the left ventricular mass. LVW/BW ratio $(\mathrm{mg} / \mathrm{g})=$ left ventricular weight $(\mathrm{mg}) /$ body weight (g) (19).

\section{Collagen Volume Fraction and Perivascular Collagen Area}

Masson staining was used to evaluate the severity of myocardial fibrosis (20). Based on Masson staining sections of myocardial tissues, CVF and PVCA were calculated by Image Pro Plus software version 6.0. Masson tri-color staining kit were purchased from KeyGEN Biotech (Nanjing, China).

\section{Immunohistochemistry of Collagen I}

Histological determinations of cardiac fibrosis were performed in $5 \mu \mathrm{m}$ thick sections of paraffin-embedded myocardial tissue. The immunochemistry was performed following the protocol. For each immunochemistry and staining, serial sections were done. The most representative image was shown. Image J software was used to quantitatively calculate the expression of Col I in myocardial tissue.

\section{Rhodamine 123}

The mitochondrial membrane potential assay in myocardial tissue was conducted by Rhodamine 123 staining. Disruption of mitochondrial potential has been shown to be featured with the decrease in Rhodamine 123 retention and fluorescence (21). The microtubes containing $1 \mathrm{~mL}$ of mitochondrial solution mixed with $10 \mu \mathrm{l}$ of rhodamine 123 dye solution were incubate at $37^{\circ} \mathrm{C}$ for $1 \mathrm{~h}$ in the dark. After washing three times with PBS, the fluorescence intensity was measured with a microplate reader.

\section{Western Blot}

The protein expression levels of Col I and Col III in myocardial tissue were evaluated using western blot, with 
glyceraldehyde-3-phosphate dehydrogenase (GAPDH) protein as the reference. The relative proteins of mitochondrial dynamics such as Mitofusin-1, Mitofusin-2 and Drp1 in myocardial tissue were also detected using western blot, with voltagedependent anion channel (VDAC) protein as the reference.
About $100 \mathrm{mg}$ of myocardial tissue were cut into small pieces and put into a pre-cooled EP tube. Total protein concentration was determined according to the instructions of the BCA kit. The supernatants were collected. Then they were separated by SDS-polyacrylamide gels and transferred to a PVDF membrane.
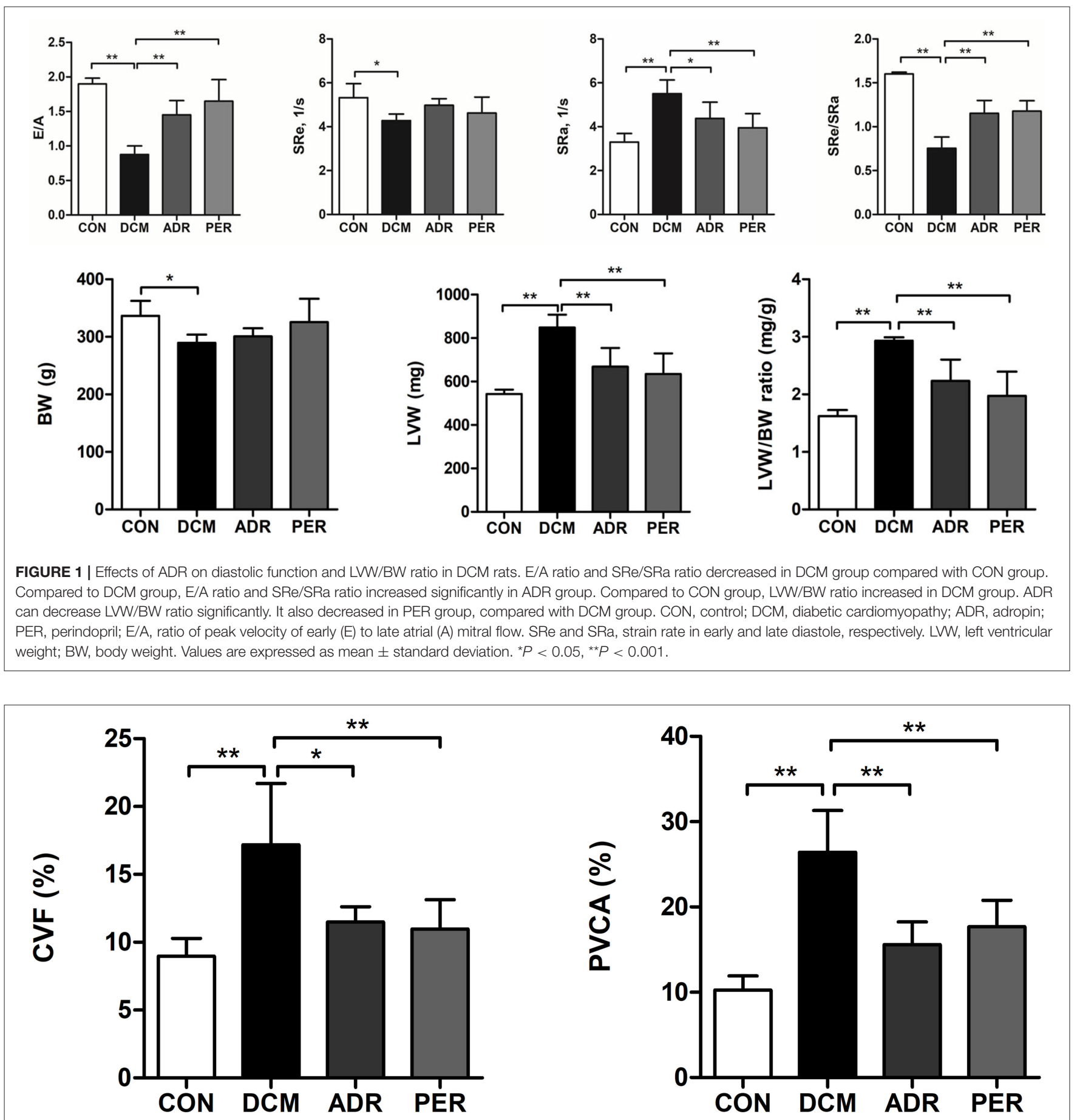

FIGURE 2 | Effects of ADR on CVF and PVCA of myocardial tissue in DCM rats. Compared to DCM group, CVF and PVCA decreased in ADR and PER group. CON, control; DCM, diabetic cardiomyopathy; ADR, adropin; PER, perindopril; CVF, collagen volume fraction; PVCA, perivascular collagen volume area. Values are expressed as mean \pm standard deviation. ${ }^{\star} P<0.05,{ }^{\star *} P<0.01$. 

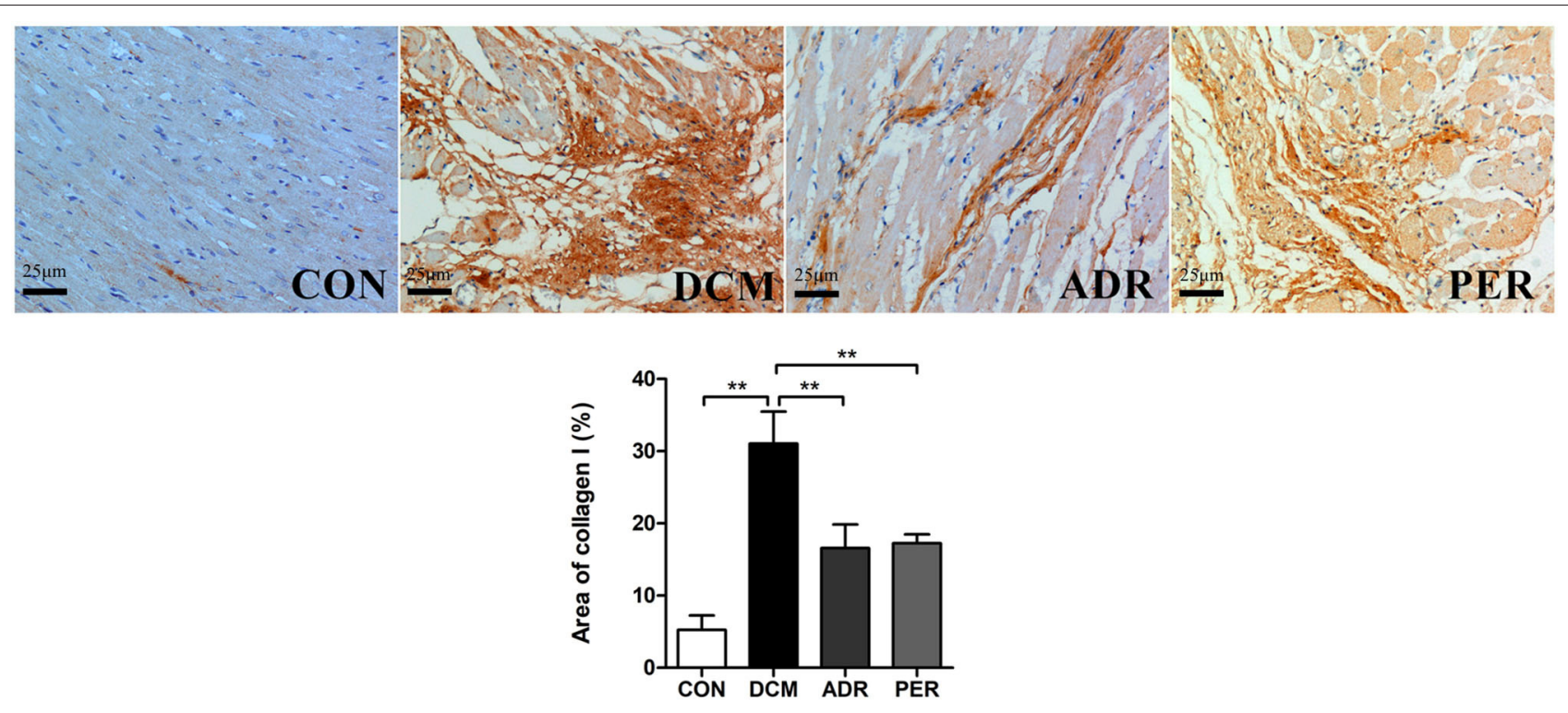

FIGURE 3 | Immunohistochemistry of Col I in myocardial tissues in DCM rats (x 200). Compared to DCM group, the color of collagen fibers in ADR group and PER group becomes lighter and the expression of Col I reduced remarkably. CON, control; DCM, diabetic cardiomyopathy; ADR, adropin; PER, perindopril. Values are expressed as mean \pm standard deviation. ${ }^{\star} P<0.05$, ${ }^{\star \star} P<0.01$.

The membranes were blocked with $5 \%$ skimmed milk powder TBST solution for $1 \mathrm{~h}$ at room temperature and incubated with primary antibodies overnight at $4{ }^{\circ} \mathrm{C}$. The remaining liquid on the membrane was washed with TBST. Images were captured with Tanon 6600 luminescence imaging workstation (Tanon Science \& Technology Co., Ltd. Shanghai, China).

\section{Statistical Analysis}

Data were presented as group mean and its standard error (mean \pm SEM). One-way analysis of variance (ANOVA) followed by the least significant difference (LSD) post hoc test was used for the statistical analysis of the difference between multiple groups. All statistical analyses were performed in SPSS 20.0 (IBM Corp., Armonk, USA).

\section{RESULTS}

\section{Effects of ADR on Diastolic Function and LVW/BW Ratio}

As shown in Figure 1, E/A ratio and SRe/SRa ratio were used to assess the diastolic function of DCM rats. LVW/BW ratio was used to assess the severity of cardiac remodeling in DCM rats. E/A ratio $(1.90 \pm 0.09$ vs. $0.89 \pm 0.12, P<0.001)$ and SRe/SRa ratio ( $1.60 \pm 0.02$ vs. $0.75 \pm 0.13, P<0.001)$ dercreased in DCM group compared with CON group. Compared to DCM group, E/A ratio $(1.43 \pm 0.18$ vs. $0.89 \pm 0.12, P=0.001)$ and SRe/SRa ratio $(1.15 \pm 0.15$ vs. $0.75 \pm 0.13, P<0.001)$ increased significantly in ADR group. LVW (543.26 $\pm 19.44 \mathrm{mg}$ vs. 849.05 $\pm 58.91 \mathrm{mg}, P<0.001)$ and LVW/BW ratio $(1.62 \pm 0.11 \mathrm{mg} / \mathrm{g}$ vs. $2.93 \pm 0.06 \mathrm{mg} / \mathrm{g}, P<0.001)$ increased in DCM group compared with CON group. Compared to $\mathrm{DCM}$ group, LVW/BW ratio decreased significantly in ADR $(2.23 \pm 0.37 \mathrm{mg} / \mathrm{g}$ vs. $2.93 \pm 0.06$ $\mathrm{mg} / \mathrm{g}, P=0.005)$ and PER group $(1.98 \pm 0.42 \mathrm{mg} / \mathrm{g}$ vs. $2.93 \pm$ $0.06 \mathrm{mg} / \mathrm{g}, P=0.001)$.

\section{Effects of ADR on CVF and PVCA of Myocardial Tissue}

Using Masson staining sections and Image Pro Plus version 6.0 software, CVF and PVCA of myocardial tissue were calculated (Figure 2). Compared to CON group, CVF (8.97 $\pm 1.31 \%$ vs. $17.18 \pm 4.53 \%, P=0.001)$ and PVCA $(10.24 \pm 1.68 \%$ vs. 26.41 $\pm 4.92 \%, P<0.001)$ in DCM group increased dramatically. CVF and PVCA in ADR group (11.49 $\pm 1.12 \%$ vs. $17.18 \pm 4.53 \%, P$ $=0.001 ; 15.58 \pm 2.67 \%$ vs. $26.41 \pm 4.92 \%, P<0.001)$ and PER group $(10.98 \pm 2.16 \%$ vs. $17.18 \pm 4.53 \%, P=0.001 ; 17.67 \pm$ $3.12 \%$ vs. $26.41 \pm 4.92 \%, P<0.001)$ decreased significantly in the comparison with DCM group.

\section{Effects of ADR on Immunohistochemistry of Col I in Myocardial Tissue}

The immunohistochemical staining of Col I in myocardial tissue was used to evaluate the severity of cardiac fibrosis (Figure 3). Collagen fibers from greenish yellow to orange red and red color as the severity of the cardiac fibrosis increased. In CON group, a small amount of Col I was scattered and the myocardial tissues arranged neatly. The Col I expression increased significantly in DCM group, showing strong positive staining in the form of flake. Quantitative calculation of the Col I expression in myocardial tissue was also made via Image J software. The Col I volume fractions in CON, DCM, ADR and PER group were $5.26 \pm 1.98 \%, 31.06 \pm 4.42 \%, 16.57 \pm 3.29 \%$, and $17.25 \pm$ $1.24 \%$, respectively. Compared with the DCM group, the color 

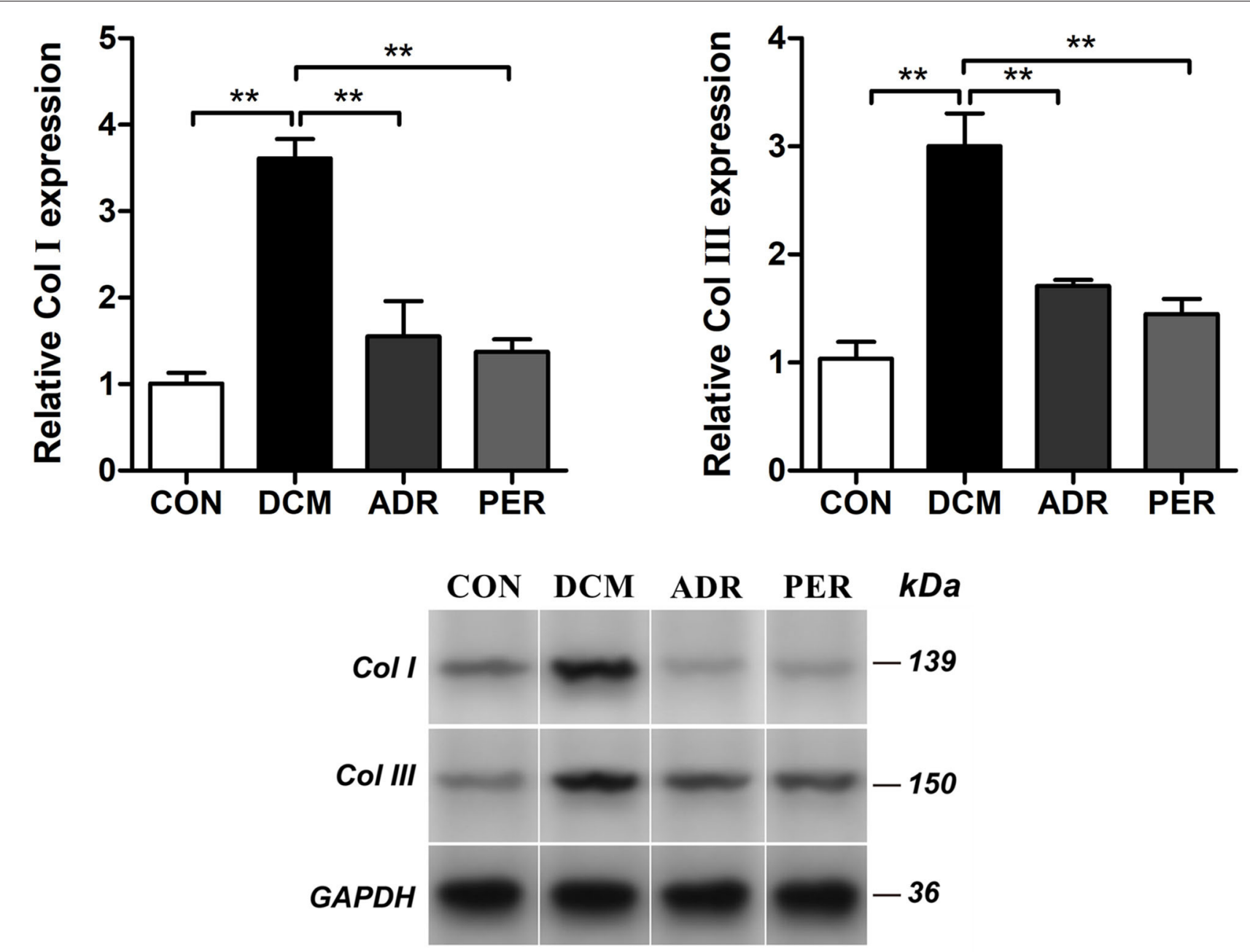

FIGURE 4 | Effects of ADR on relative expression of Col I and Col III in myocardial tissue in DCM rats. Compared to DCM group, the relative expression of Col I and Col III proteins decreased in ADR and PER group. CON, control; DCM, diabetic cardiomyopathy; ADR, adropin; PER, perindopril; Col, collagen. Values are expressed as mean \pm standard deviation. ${ }^{*} P<0.05,{ }^{* *} P<0.01$.

of collagen fibers in ADR group and PER group becomes lighter and the expression of Col I reduced remarkably $(P<0.001)$.

\section{Effects of ADR on Relative Expression of Col I and Col III Proteins}

The expression of Col I and Col III proteins in myocardial tissue were detected with Western Blot (Figure 4). Compared to CON group, the relative expression of Col I $(1.01 \pm 0.13$ vs. $3.61 \pm 0.23$, $P<0.001)$ and Col III $(1.03 \pm 0.15$ vs. $3.00 \pm 0.30, P<0.001)$ increased dramatically in DCM group. The relative expression of Col I decreased in ADR $(1.55 \pm 0.41$ vs. $3.61 \pm 0.23, P<0.001)$ and PER $(1.37 \pm 0.15$ vs. $3.61 \pm 0.23, P<0.001)$ group in the comparison with DCM group. Besides, compared to DCM group, the relative expression of $\mathrm{Col}$ III also reduced in $\mathrm{ADR}(1.71 \pm$ 0.06 vs. $3.00 \pm 0.30, P<0.001)$ and PER $(1.45 \pm 0.14$ vs. $3.00 \pm$ $0.30, P<0.001)$ group.

\section{Effects of ADR on the Fluorescence of Rhodamine 123}

Alteration in the mitochondrial transmembrane potential in myocardial tissue was determined by Rhodamine 123 staining. Disruption of the mitochondrial membrane followed by the loss in the mitochondrial membrane potential is a key step in the mitochondrial apoptotic pathway. As shown in Figure 5, the fluorescence of Rhodamine 123 in DCM group (58.97 \pm 4.19 vs. $36.28 \pm 1.04, P<0.001$ ) decreased significantly in the comparison with CON group. However, the fluorescence of Rhodamine 123 in $\operatorname{ADR}(48.10 \pm 3.58$ vs. $36.28 \pm 1.04, P<$ $0.001)$ and PER group $(48.19 \pm 3.85$ vs. $36.28 \pm 1.04, P<0.001)$ increased in the comparison with DCM group.

\section{Effects of ADR on the Relative Expression of Proteins in Mitochondrial Dynamics}

The relative proteins of mitochondrial dynamics in myocardial tissue were detected with Western Blot (Figure 6). Compared to 
CON group, the relative expression of Mitofusin-1 $(1.00 \pm 0.12$ vs. $0.36 \pm 0.08, P<0.001)$ and Mitofusin-2 $(1.00 \pm 0.13$ vs. 0.41 $\pm 0.04, P<0.001)$ proteins decreased dramatically. The relative expression of Mitofusin-1 and Mitofusin-2 proteins increased in $\operatorname{ADR}(0.83 \pm 0.14$ vs. $0.36 \pm 0.08, P=0.001 ; 0.85 \pm 0.11$ vs. 0.41 $\pm 0.04, P=0.001)$ and PER $(0.86 \pm 0.07$ vs. $0.36 \pm 0.08, P<$ $0.001 ; 0.82 \pm 0.12$ vs. $0.41 \pm 0.04, P=0.001)$ group, respectively, in comparison with DCM group. Compared to CON group, the relative expression of Drp1 protein $(1.00 \pm 0.11$ vs. $2.89 \pm 0.52$, $P<0.001)$ increased significantly. However, it decreased in ADR ( $1.42 \pm 0.36$ vs. $2.89 \pm 0.52, P=0.001)$ or PER ( $1.58 \pm 0.33$ vs. $2.89 \pm 0.52, P=0.002)$ group in comparison with DCM group.

\section{DISCUSSION}

DCM is one of the most important complications of diabetes mellitus. Mitochondrial dysfunction is an important factor in the occurrence of DCM. The imbalance of mitochondrial dynamics is generally considered the cause of DCM. In the diabetic condition, total adenosine triphosphate was generated from fatty acid oxidation instead of glucose, which generates more reactive oxygen species and disrupts the oxidative phosphorylation process (4). It may cause calcium overload, induce the opening of mitochondrial permeability transition pore and lead to mitochondrial dysfunction (22).

In the present study, we found that $\mathrm{ADR}$ reduced $\mathrm{LVW} / \mathrm{BW}$ ratio, E/A ratio, SRe/SRa ratio, $\mathrm{CVF}, \mathrm{PVCA}$ and the relative expression of Col I and Col III proteins, indicating that ADR can alleviate myocardial fibrosis and improve diastolic function in DCM rats. Until now there are no published articles discussing the effect of ADR on myocardial fibrosis and cardiac remodeling in DCM. However, ADR was proven to protect against liver fibrosis in a previous study. The authors measured the serum ADR levels, liver injury and oxidative stress in diet-induced non-alcoholic steatohepatitis mice. ADR knockout mice and palmitate treated primary hepatic cells were used to investigate the influence of ADR on liver injury. The results indicated that the treatment with ADR alleviated hepatocyte injury by upregulating the expression of Gclc, Gclm, and Gpx1 in a manner dependent on Nrf2 transcriptional activity and by increasing the glutathione levels (23). Therefore, the present study is the first article to report that $\mathrm{ADR}$ may reduce myocardial fibrosis in DCM rats. Moreover, based on the previous related researches on other disease models $(12,23)$, ADR seems to be effective in improving organ injury and fibrosis.

Moreover, our study also explored the role of mitochondrial dynamics in the process of ADR protecting against myocardial fibrosis in DCM rats. Mitochondria is extremely important for energy supply of heart. As we know, the heart requires large amounts of high-energy phosphates and accounts for $\sim 8 \%$ of the total adenosine triphosphate consumption of the body. The majority of adenosine triphosphate is regenerated in the mitochondria via oxidative phosphorylation $(5,24)$. Mitochondria are highly dynamic organelles undergoing coordinated cycles of fission and fusion, which referred as mitochondrial dynamics (25). In mammalian cells, the

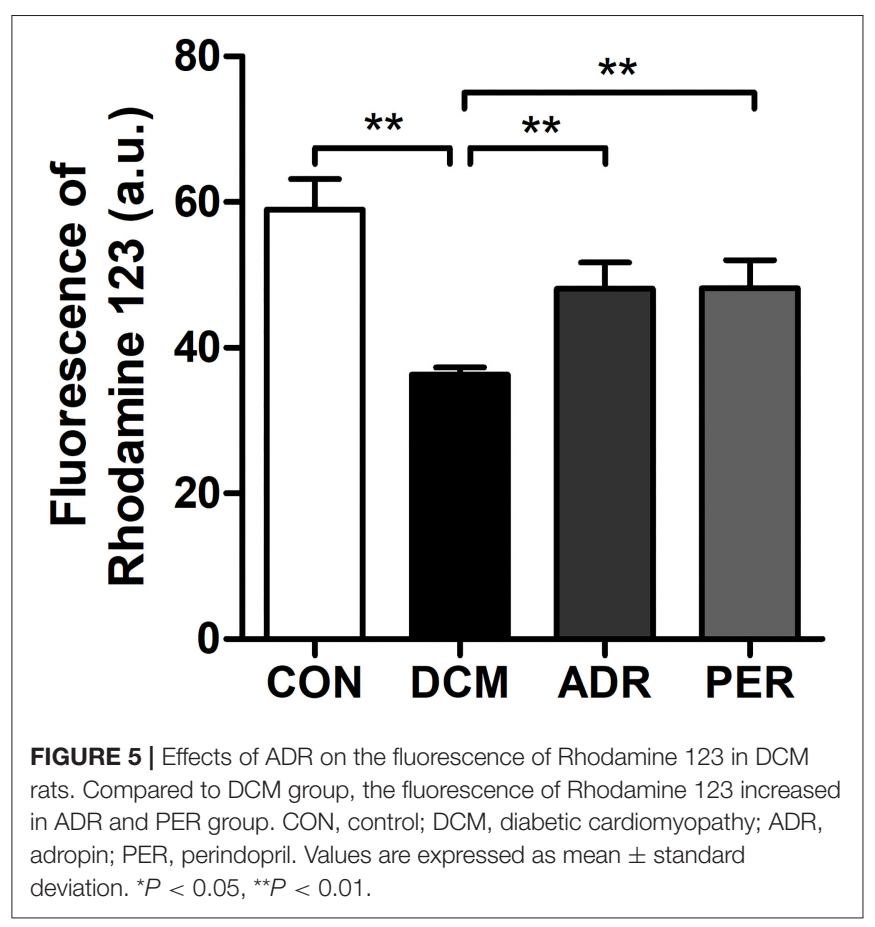

primary regulators of mitochondrial fusion are dynamin-related mitofusins (mitofusin-1 and mitofusin-2) and optic atrophy protein 1 (OPA1). On the other side, mitochondrial fission 1 protein and the dynamin-related protein 1 (DRP1) are involved in mitochondrial fission. The balance of mitochondrial fusion and fission has been recognized as critical processes in the health of mitochondria and cells $(5,26)$.

The results of this study found that the balance of mitochondrial fusion and fission has been broken in DCM rats compared with $\mathrm{CON}$ rats via detecting mitochondrial dynamics related proteins. Besides, ADR can upregulate the relative expression of Mitofusin-1 and Mitofusin-2 proteins and downregulate the expression of Drp1 protein in DCM rats. Thapa et al. $(27,28)$ had also conducted a series of studies concerning the effect of ADR in cardiac cells and pre-diabetic obese mice. In vitro experiments, the stimulation of cultured cardiac cells with ADR may lead to decreased expression of the pyruvate dehydrogenase negative regulator PDK4, which reduced inhibitory $\mathrm{PDH}$ phosphorylation. In vivo study, cardiac glucose oxidation reduced in high fat diet animals. Acute ADR treatment increased cardiac glucose oxidation. ADR can modulate the expression of the mitochondrial acetyltransferase enzyme GCN5L1, altering the activity of fuel metabolism enzymes to favor glucose utilization. These findings suggest that $\mathrm{ADR}$ may be a key regulator of fuel substrate utilization in the heart and may provide a future therapeutic avenue in the treatment of DCM.

There are some limitations in this study. Firstly, much of the current knowledge regarding DCM is the result of studies performed using adult streptozotocin-induced diabetic rodents. However, the mechanism of DCM in this model may be 

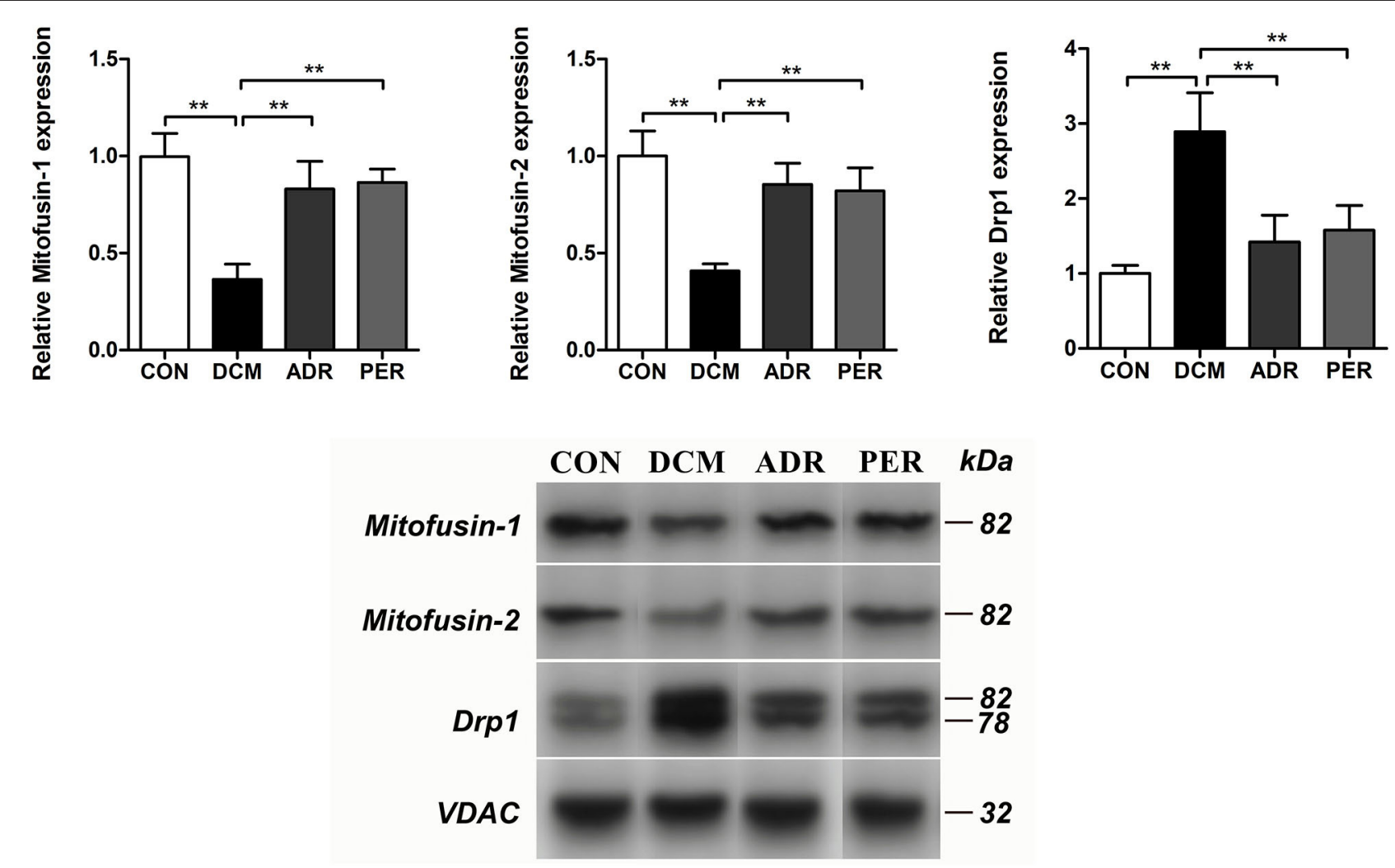

FIGURE 6 | Effects of ADR on the relative proteins of mitochondrial dynamics in DCM rats. Compared to DCM group, the relative expression of Mitofusin-1 and Mitofusin-2 proteins increased in ADR and PER group. The relative expression of Drp1 protein decreased in ADR and PER group in comparison with DCM group. CON, control; DCM, diabetic cardiomyopathy; ADR, adropin; PER, perindopril. Values are expressed as mean \pm standard deviation. ${ }^{\star} P<0.05,{ }^{\star \star} P<0.01$.

related the direct actions of streptozotocin on muscle fibers independent of the diabetic phenotype. Therefore, future studies should be repeated in alternative models of DCM, such as Ins2 (+/Akita) mouse and high fat diet-fed models. Secondly, this is a preliminary study on the effect of ADR in DCM rats. Even though several mitochondrial dynamics related proteins had been detected, other signaling pathways or parameters related to energy metabolism and mitochondrial respiration have not been tested. Besides, this study did not report the results of in vitro experiments. The result mainly implied the phenomenon. Further study should be conducted to observe the effects of ADR on different specific cells in myocardial tissue. Moreover, this study did not explore the involvement of mitochondrial apoptosis in DCM. Therefore, further studies are needed in the future to confirm the mechanism of ADR in DCM.

\section{CONCLUSIONS}

In summary, the present study demonstrated ADR could alleviate myocardial fibrosis and improve diastolic function in DCM rats. Our present study is the first to describe the effect of ADR on myocardial fibrosis in DCM rats. ADR may be a putative candidate for the treatment of DCM.

\section{DATA AVAILABILITY STATEMENT}

The raw data supporting the conclusions of this article will be made available by the authors, without undue reservation.

\section{ETHICS STATEMENT}

The animal study was reviewed and approved by Ethics Committee of North Sichuan Medical College.

\section{AUTHOR CONTRIBUTIONS}

ML and JA: conception and design. ML: administrative support. ZS and JA: provision of study materials or patients. ZS, JA, and KT: collection and assembly of data. JA, KT, ZL, and YH: data analysis and interpretation. ML, JA, and $\mathrm{YH}$ : manuscript writing. All authors contributed to the article and approved the submitted version.

\section{FUNDING}

This study was supported by the Research Project of Affiliated Hospital of North Sichuan Medical College (No. 2020ZD014). 


\section{REFERENCES}

1. Paolillo S, Marsico F, Prastaro M, Renga F, Esposito L, De Martino F, et al. Diabetic cardiomyopathy: definition, diagnosis, and therapeutic implications. Heart Fail Clin. (2019) 15:341-7. doi: 10.1016/j.hfc.2019.02.003

2. Varma U, Koutsifeli P, Benson VL, Mellor KM, Delbridge L. Molecular mechanisms of cardiac pathology in diabetes experimental insights. Biochim Biophys Acta Mol Basis Dis. (2018) 1864:1949-59. doi: 10.1016/j.bbadis.2017.10.035

3. Yue E, Yu Y, Wang X, Liu B, Bai Y, Yang B. Anthocyanin protects cardiac function and cardiac fibroblasts from high-glucose induced inflammation and myocardial fibrosis by inhibiting IL-17. Front Pharmacol. (2020) 11:593633. doi: 10.3389/fphar.2020.593633

4. Jubaidi FF, Zainalabidin S, Mariappan V, Budin SB. Mitochondrial dysfunction in diabetic cardiomyopathy: the possible therapeutic roles of phenolic acids. Int J Mol Sci. (2020) 21:6043. doi: 10.3390/ijms21176043

5. Gollmer J, Zirlik A, Bugger H. Mitochondrial mechanisms in diabetic cardiomyopathy. Diabetes Metab J. (2020) 44:3353. doi: $10.4093 / \mathrm{dmj} .2019 .0185$

6. Ticinovic KT, Milicevic T, Novak A, Vilovic M, Bozic J. Adropin potential link in cardiovascular protection for obese male type 2 diabetes mellitus patients treated with liraglutide. Acta Clin Croat. (2020) 59:34450. doi: 10.20471/acc.2020.59.02.19

7. Zheng J, Liu M, Chen L, Yin F, Zhu X, Gou J, et al. Association between serum adropin level and coronary artery disease: a systematic review and metaanalysis. Cardiovasc Diagn Ther. (2019) 9:1-7. doi: 10.21037/cdt.2018.07.09

8. Jasaszwili M, Billert M, Strowski MZ, Nowak KW, Skrzypski M. Adropin as a fat-burning hormone with multiple functions-review of a decade of research. Molecules. (2020) 25:549. doi: 10.3390/molecules25030549

9. Maciorkowska M, Musialowska D, Malyszko J. Adropin and irisin in arterial hypertension, diabetes mellitus and chronic kidney disease. Adv Clin Exp Med. (2019) 28:1571-5. doi: 10.17219/acem/104551

10. Kolben Y, Weksler-Zangen S, Ilan Y. Adropin as a potential mediator of the metabolic system-autonomic nervous system-chronobiology axis: implementing a personalized signature-based platform for chronotherapy. Obes Rev. (2021) 22:e13108. doi: 10.1111/obr.13108

11. Altamimi TR, Gao S, Karwi QG, Fukushima A, Rawat S, Wagg $\mathrm{CS}$, et al. Adropin regulates cardiac energy metabolism and improves cardiac function and efficiency. Metabolism. (2019) 98:37-48. doi: 10.1016/j.metabol.2019.06.005

12. Wu L, Fang J, Yuan X, Xiong C, Chen L. Adropin reduces hypoxia/reoxygenation-induced myocardial injury via the reperfusion injury salvage kinase pathway. Exp Ther Med. (2019) 18:330714. doi: 10.3892/etm.2019.7937

13. Arkadievich OD. Metabolic markers of myocardium insulin resistance in dogs with heart failure. Open Vet J. (2021) 10:363-70. doi: 10.4314/ovj.v10i4.2

14. Mushala B, Scott I. Adropin: a hepatokine modulator of vascular function and cardiac fuel metabolism. Am J Physiol Heart Circ Physiol. (2021) 320:H23844. doi: 10.1152/ajpheart.00449.2020

15. Akcilar R, Emel KF, Simsek H, Akcilar A, Bayat Z, Ece E, et al. The effect of adropin on lipid and glucose metabolism in rats with hyperlipidemia. Iran J Basic Med Sci. (2016) 19:245-51. doi: 10.22038/IJBMS.2016.6642

16. Abd ES, Fayed HM. Targeting AngII/AT1R signaling pathway by perindopril inhibits ongoing liver fibrosis in rat. J Tissue Eng Regen Med. (2019) 13:213141. doi: $10.1002 /$ term. 2940
17. Luo J, Yan D, Li S, Liu S, Zeng F, Cheung CW, et al. Allopurinol reduces oxidative stress and activates Nrf2/p62 to attenuate diabetic cardiomyopathy in rats. J Cell Mol Med. (2020) 24:1760-73. doi: 10.1111/jcmm.14870

18. Youssef ME, Abdelrazek HM, Moustafa YM. Cardioprotective role of GTS21 by attenuating the TLR4/NF-kappaB pathway in streptozotocin-induced diabetic cardiomyopathy in rats. Naunyn Schmiedebergs Arch Pharmacol. (2021) 394:11-31. doi: 10.1007/s00210-020-01957-4

19. Jin $\mathrm{X}, \mathrm{Yu} \mathrm{LL}, \mathrm{Yu} \mathrm{CX}$. Effect of miR-9 on myocardial fibrosis in rats via TGF-beta1/Smads signaling pathway. Eur Rev Med Pharmacol Sci. (2019) 23: 7083-8. doi: 10.26355/eurrev_201908_18752

20. Chen P, Qiao D, Liu X. Effects and mechanism of SO2 inhalation on rat myocardial collagen fibers. Med Sci Monit. (2018) 24:16629. doi: 10.12659/MSM.904796

21. Khan I, Khan F, Farooqui A, Ansari IA. Andrographolide exhibits anticancer potential against human colon cancer cells by inducing cell cycle arrest and programmed cell death via augmentation of intracellular reactive oxygen species level. Nutr Cancer. (2018) 70:787-803. doi: 10.1080/01635581.2018.1470649

22. Nguyen BY, Ruiz-Velasco A, Bui T, Collins L, Wang X, Liu W. Mitochondrial function in the heart: the insight into mechanisms and therapeutic potentials. Br J Pharmacol. (2019) 176:4302-18. doi: 10.1111/bph.14431

23. Chen $X$, Xue $H$, Fang $W$, Chen $K$, Chen $S$, Yang $W$, et al. Adropin protects against liver injury in nonalcoholic steatohepatitis via the Nrf2 mediated antioxidant capacity. Redox Biol. (2019) 21:101068. doi: 10.1016/j.redox.2018.101068

24. Whitley BN, Engelhart EA, Hoppins S. Mitochondrial dynamics and their potential as a therapeutic target. Mitochondrion. (2019) 49:26983. doi: $10.1016 /$ j.mito.2019.06.002

25. Tilokani L, Nagashima S, Paupe V, Prudent J. Mitochondrial dynamics: overview of molecular mechanisms. Essays Biochem. (2018) 62:34160. doi: 10.1042/EBC20170104

26. Chan DC. Mitochondrial dynamics and its involvement in disease. Annu Rev Pathol. (2020) 15:235-59. doi: 10.1146/annurev-pathmechdis-012419-0 32711

27. Thapa D, Stoner MW, Zhang M, Xie B, Manning JR, Guimaraes D, et al. Adropin regulates pyruvate dehydrogenase in cardiac cells via a novel GPCR-MAPK-PDK4 signaling pathway. Redox Biol. (2018) 18:25-32. doi: 10.1016/j.redox.2018. 06.003

28. Thapa D, Xie B, Zhang M, Stoner MW, Manning JR, Huckestein BR, et al. Adropin treatment restores cardiac glucose oxidation in pre-diabetic obese mice. J Mol Cell Cardiol. (2019) 129:174-8. doi: 10.1016/j.yjmcc.2019. 02.012

Conflict of Interest: The authors declare that the research was conducted in the absence of any commercial or financial relationships that could be construed as a potential conflict of interest.

Copyright (C) 2021 Liu, Ai, Shuai, Tang, Li and Huang. This is an open-access article distributed under the terms of the Creative Commons Attribution License (CC BY). The use, distribution or reproduction in other forums is permitted, provided the original author(s) and the copyright owner(s) are credited and that the original publication in this journal is cited, in accordance with accepted academic practice. No use, distribution or reproduction is permitted which does not comply with these terms. 